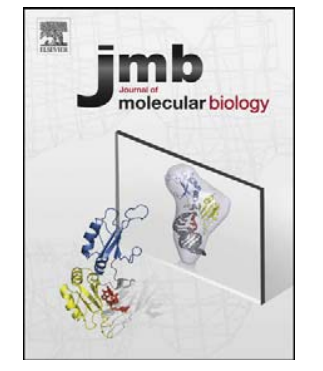

\title{
Structural Insights into Nonspecific Binding of DNA by TrmBL2, an Archaeal Chromatin Protein
}

\author{
Misbha Ud Din Ahmad ${ }^{1}$, Ingrid Waege ${ }^{2}$, Winfried Hausner ${ }^{2}$, Michael Thomm ${ }^{2}$, \\ Winfried Boos ${ }^{1}$, Kay Diederichs ${ }^{1}$ and Wolfram Welte ${ }^{1}$ \\ 1 - University of Konstanz Department of Biology, Universitätsstrasse 10, 78456 Konstanz, Germany \\ 2 - University Regensburg Lehrstuhl für Mikrobiologie, Universitätsstrasse 31, 93053 Regensburg, Germany
}

Correspondence to Wolfram Welte: wolfram.welte@uni-konstanz.de

\begin{abstract}
The crystal structure of TrmBL2 from the archaeon Pyrococcus furiosus shows an association of two pseudosymmetric dimers. The dimers follow the prototypical design of known bacterial repressors with two helix-turn-helix $(\mathrm{HTH})$ domains binding to successive major grooves of the DNA. However, in TrmBL2, the two dimers are arranged at a mutual displacement of approximately 2 bp so that they associate with the DNA along the double-helical axis at an angle of approximately $80^{\circ}$.

While the deoxyribose phosphate groups of the double-stranded DNA (dsDNA) used for co-crystallization are clearly seen in the electron density map, most of the nucleobases are averaged out. Refinement required to assume a superposition of at least three mutually displaced dsDNAs. The HTH domains interact primarily with the deoxyribose phosphate groups and polar interactions with the nucleobases are almost absent.

This hitherto unseen mode of DNA binding by TrmBL2 seems to arise from nonoptimal protein-DNA contacts made by its four HTH domains resulting in a low-affinity, nonspecific binding to DNA.
\end{abstract}

\section{Introduction}

The first member of the TrmB family of transcriptional regulators was discovered in the hyperthermophilic archaea Thermococcus litoralis/Pyrococcus furiosus that controlled the transcription of the genes encoding the $A B C$ transporter for trehalose and maltose, the TM system, with maltose and trehalose as inducers. The surprising feature of this regulator was its ability to also control the $A B C$ transporter for maltodextrins, the MD system, with maltotriose and sucrose as inducers [1]. The crystal structure of TrmB [2] revealed an extended winged helix-turn-helix (ewHTH) motif suggesting the binding of the dimeric TrmB at the palindromic sequence in the promoter region of the TM operon.

The early search for homologs of TrmB within four members of the Thermococcales showed a series of five proteins named TrmBL1, TrmBL2, TrmBL3 and TrmBL4 [3,4]. These sequences showed a considerable degree of sequence identity at the $\mathrm{N}$-terminus overlapping the ewHTH motif seen in the crystal structure of TrmB. Apart from TrmBL2, not all TrmB members are present in all four Thermococcales. $A$ recent review discusses the now large TrmB family recognized in the archaea [3,5].

The ortholog of TrmBL1 in Thermococcus kodakarensis, named Tgr (Thermococcales glycolytic regulator), was found to prominently recognize the palindromic TGM (Thermococcales glycolytic motif) sequence $[6,7]$. Depending on its position within the respective promoter, Tgr binds to it, acting as an activator when present upstream of BRE/TATA box of genes encoding gluconeogenic proteins but as a repressor when positioned downstream of the BRE/ TATA box of genes encoding glycolytic genes. TrmBL1 of $P$. furiosus showing $67 \%$ sequence identity to Tgr and exhibits the same function as Tgr [3].

The data obtained mainly with TrmB and Tgr/ TrmBL1 characterized them as specific transcriptional regulators recognizing their specific target DNA and being controlled by binding of different sugars.

Sequence alignment of the TrmB family of proteins revealed that TrmBL2 lacks the $\mathrm{C}$-terminal sugar- 
binding subdomain present in other TrmB orthologs $[3,5]$. Nevertheless, when tested by band shift analysis and run-off transcription assays, it was found that TrmBL2 was able to recognize the MD promoter but no obvious pattern with respect to other TGM-containing promoters could be detected, neither could a specific DNA sequence motif for TrmBL2 be identified [3,5].

Recently, a novel function of TK0471, the T. kodakarensis ortholog of TrmBL2, came to light. Apart from acting as a transcriptional regulator, it was observed that TrmBL2 associates nonspecifically to the chromatin forming thick fibrous structures [8]. The appearance of these thick fibrous structures only in the log phase suggests that TrmBL2 exerts its effect on the chromatin in a growth-phase-dependent manner. The observation that the thick fibrous structure is lost in TrmBL2 deletion strains with a concomitant increase in expression of a large number of genes pointed out to its dual role of being a global repressor and a DNA structuring protein.

A more recent study about the role of TrmBL2 in archaeal chromosome organization shows that it counteracts the role of histones in DNA packaging and actively competes with histones for DNA binding. It was also shown that TrmBL2 exhibits concentration-dependent, high- and low-affinity DNA binding modes that are influenced by the salt concentration of the medium. The study also demonstrates that nonspecific interactions of TrmBL2 with DNA backbone contribute significantly to the TrmBL2-DNA binding energy [9]. Together with archaeal histones [10] and Alba (acetylation lowers binding affinity) [10,11], TK0471/TrmBL2 is now considered to be a member of chromosome structuring proteins in archaea [12].

TrmBL2 does not show significant sequence or structural similarity to the established DNA binding proteins such as Alba or archaeal histones. However, the nonspecific association with DNA suggests a design of its ewHTH motif in DNA interaction that is different from the specific recognition of an operator sequence seen in transcriptional regulators. Here we present the crystal structure of TrmBL2 from $P$. furiosus alone and in complex with DNA revealing an as yet unknown mode of binding that suggests a structural explanation for a mechanism of nonspecific binding.

\section{Results}

\section{TrmBL2 was crystallized with and without dsDNA}

Full-length TrmBL2, 264 residues, was cloned and expressed in Escherichia coli BL21 DE3 cells (named ecTrmBL2 hereafter). Purification was possible without a tag because of the extreme heat stability of the protein that allowed removal of contaminating proteins by incubation at $80^{\circ} \mathrm{C}$ for 20 min (see Materials and Methods). In the final gel-permeation chromatography, the pure protein eluted at a volume corresponding to an ecTrmBL2 dimer (see Supplementary Fig. 1). Another TrmBL2 construct with a C-terminal hexa-histidine tag was overexpressed in engineered $P$. furiosus cells and purified by NiNTA chromatography (named pfTrmBL2 hereafter). The elution volume from the final gel-permeation chromatography was in accord with a pfTrmBL2 tetramer (see Supplementary Fig. 1).

ecTrmBL2-DNA complexes were formed by mixing the protein with TGM17 or TGM19 double-stranded DNA (dsDNA) in a 1:3.4 molar ratio prior to setting up crystallization trials. Crystals grew at $18{ }^{\circ} \mathrm{C}$ and reached a maximum size after 4 weeks of incubation. Prior to data collection, the crystals were mounted, flash-cooled and stored in liquid nitrogen. Phasing was achieved with a selenomethionine derivative of ecTrmBL2. For details of data collection, see Table 1.

\section{Description of the Crystal Structures}

\section{Overall structure and similarities with TrmB}

TrmBL2, alone or in complex with TGM19 or TGM17 DNA, exhibits the same tetrameric structure in the asymmetric unit of all crystals (See Fig. 1 for the ecTrmBL2-TGM19 complex and Fig. 2b and c for a detailed representation of the various domains). A 2-fold noncrystallographic symmetry (NCS) relates both, the two protein dimers (chains $A C$ and chains $B D$, respectively) and the two strands of the DNA with each other. The TGM DNA is bent as seen when the symmetry axis is oriented vertically (see Fig. 1b). This indicates symmetrical interactions of both TrmBL2 dimers with the DNA. The TrmBL2 dimer shares the general order of domains with the TrmB dimer [2]. The $\mathrm{N}$-terminal ewHTH domains of both proteins can be superimposed with an RMSD of $1.83 \AA$ (calculated by SSM superpose [13] in Coot). A dimerization helix (a4) and a C-terminal domain (termed CTD in TrmBL2 and EBD in TrmB) follow in both proteins. In both proteins, the long dimerization helix is amphipathic and forms a coiled coil with an antiparallel arranged helix from another molecule (see Fig. 2b). Superposition with the TrmB dimerization helix is best (RMSD, $0.82 \AA$ ) when the latter is in register with the $\mathrm{N}$-terminal end of the TrmBL2 helix.

\section{The two TrmBL2 dimers and their interactions}

As in TrmB, in TrmBL2, the ewHTH domains and the dimerization helices are related by a 2-fold rotational symmetry. In TrmBL2, they superimpose 
Table 1. Data collection and refinement statistics

\begin{tabular}{|c|c|c|c|c|c|}
\hline & $\begin{array}{c}\text { ecTrmBL2- } \\
\text { TGM19 }\end{array}$ & $\begin{array}{c}\text { ecTrmBL2- } \\
\text { TGM17 }\end{array}$ & $\begin{array}{l}\text { ecTrmBL2- } \\
\text { TGM17 }\end{array}$ & pfTrmBL2 & ecTrmBL2-TGM17 peak \\
\hline \multicolumn{6}{|l|}{ Data collection } \\
\hline Wavelength $(\AA)$ & 1.00 & 1.00 & 1.00 & 1.00 & 0.97942 \\
\hline Resolution range $(\AA ̊)$ & $502.5(2.592 .5)^{a}$ & $502.4(2.492 .4)^{a}$ & $503.2(3.313 .2)^{a}$ & $503.0(3.113 .0)^{a}$ & $502.7\left(\begin{array}{lll}2.78 & 2.7)^{a}\end{array}\right.$ \\
\hline Space group & $P 2_{1}$ & $P 2_{1}$ & $P 2_{1} 2_{1} 2_{1}$ & $P 22_{1} 2_{1}$ & $P 2_{1}$ \\
\hline \multicolumn{6}{|l|}{ Unit cell parameters } \\
\hline$a(\AA)$ & 83.69 & 95.79 & 58.76 & 63.51 & 97.18 \\
\hline$b(A)$ & 105.79 & 58.67 & 154.37 & 83.29 & 59.50 \\
\hline$c(A)$ & 93.23 & 143.07 & 176.12 & 235.14 & 143.87 \\
\hline$\beta\left(^{\circ}\right)$ & 96.75 & 92.82 & 90 & 90 & 92.45 \\
\hline Multiplicity & $3.4(3.4)$ & $6.5(5.5)$ & $13.1(12.8)$ & $6.3(6.6)$ & $12.4(10.6)$ \\
\hline Completeness (\%) & $100(100)$ & $99(96)$ & $100(98)$ & $99(100)$ & $99(88)$ \\
\hline Mean $/ / \sigma$ & $7.84(0.41)$ & $8.18(0.69)$ & $7.8(0.99)$ & $9.96(0.6)$ & $9.54(0.62)$ \\
\hline$R_{\text {meas }}$ & $0.178(3.63)$ & $0.15(2.29)$ & $0.39(2.96)$ & $0.1346(2.89)$ & $0.2547(3.55)$ \\
\hline$C C_{1 / 2}$ & $0.995(0.14)$ & 0.996 (0.369) & $0.995(0.408)$ & $0.999(0.312)$ & $0.996(0.234)$ \\
\hline \multicolumn{6}{|l|}{ Refinement } \\
\hline Resolution range $(\AA ̊)$ & $502.5(2.532 .5)$ & $502.4(2.442 .4)$ & 503.2 (3.26 3.2) & $503.0(3.06 \quad 3.0)$ & \\
\hline Number of reflections & 55,705 & 62,270 & 27,265 & 25,446 & \\
\hline$R_{\text {work }}$ & 0.2242 & 0.2336 & 0.2546 & 0.2326 & \\
\hline$R_{\text {free }}$ & 0.2720 & 0.2828 & 0.2988 & 0.2888 & \\
\hline No. of amino acid residues & 1052 & 1045 & 1039 & 1038 & \\
\hline No. of nucleic acid residues & 50 & 42 & 42 & & \\
\hline No. of heteroatoms & 66 & 28 & 6 & 2 & \\
\hline RMS bonds $(\AA)$ & 0.006 & 0.002 & 0.005 & 0.005 & \\
\hline RMS angles $\left({ }^{\circ}\right)$ & 0.99 & 0.55 & 0.63 & 1.06 & \\
\hline Ramachandran favored (\%) & 94.5 & 96.6 & 95.7 & 94 & \\
\hline Ramachandran allowed (\%) & 5 & 3.3 & 4 & 5.6 & \\
\hline Ramachandran outliers (\%) & 0.5 & 0.1 & 0.3 & 0.4 & \\
\hline \multicolumn{6}{|l|}{ Average $B$-factor $\left(\AA^{2}\right)$} \\
\hline Protein & 89 & 79 & 82 & 134 & \\
\hline DNA & 98 & 96 & 103 & & \\
\hline
\end{tabular}

a Values in parenthesis are for the highest-resolution shell.

with an RMSD of $0.81 \AA$ (calculated by $\mathrm{C}^{\alpha} \mathrm{LSQ}$ superposition in Coot [14] between residues 2 and 113 of chains $A$ and $C$ ). Apart from this, the proteins differ in several ways. The TrmBL2 dimer does not exhibit a 2-fold symmetry due to distinctly different conformations of the loop regions of chains $C$ and $A$ resulting in different orientations of the two CTDs (see Fig. 2d).

The asymmetry created by the different orientations of the CTDs in the AC dimer allows the distal ewHTH of chain $A$ to form an extensive interface with the CTD of the other dimer chain C (buried surface area, $1383 \AA^{2}$ ), whereas the interface between the proximal ewHTH of chain $C$ and the CTD of chain $A$ is much smaller (buried surface area, $851 \AA^{2}$ ) (Fig. 2c). The same holds true for the other NCSrelated dimer. Altogether, the buried surfaces in the $\mathrm{AC}$ dimer and in the $\mathrm{BD}$ dimer are $2844 \AA^{2}$ and $2804 \AA^{2}$, respectively.

One of the two TrmBL2 conformations can be superimposed on TrmB so that the CTD of the former and the $\mathrm{N}$-terminal EBD subdomain of the latter correspond to each other (Fig. 3). The CTD of TrmBL2 can be superimposed on the N-terminal subdomain of the TrmB EBD with an RMSD of
2.07 A. Compared to TrmB, the dimerization helix $a 4$ of TrmBL2 is longer by approximately two turns. Furthermore, the CTD matches only the N-terminal EBD subdomain of TrmB but lacks the sugar-binding $C$-terminal EBD subdomain seen in the latter.

As in other well-known dimers of bacterial DNA binding proteins with helix-turn-helix domains [15-18], the ecTrmBL2 ewHTH domains of the dimer contact two successive major grooves in a 2 -fold symmetrical arrangement that is also shared by the dimerization helices (see Fig. 2c).

In the ecTrmBL2 crystal structure, there are two such dimers, and the base pairs with which the recognition helices $\alpha 3$ of their ewHTH domains pairs interact are shifted by two with respect to each other. This causes a rotation by about $80^{\circ}$ of the two dimers with respect to an approximated DNA axis (see Fig. 1c). This offset of the $A C$ and the BD dimers places the ewHTH domains of chains $A$ and $B$ at the distal ends of the tetramer whereas those of chains $\mathrm{C}$ and D occupy the proximal positions (Fig. 1a). The protein-protein interactions of the two TrmBL2 dimers allowing for this shift are promoted by contacts of the CTDs of chain $C$ from the AC dimer and chain $B$ from the BD dimer that differ in their 


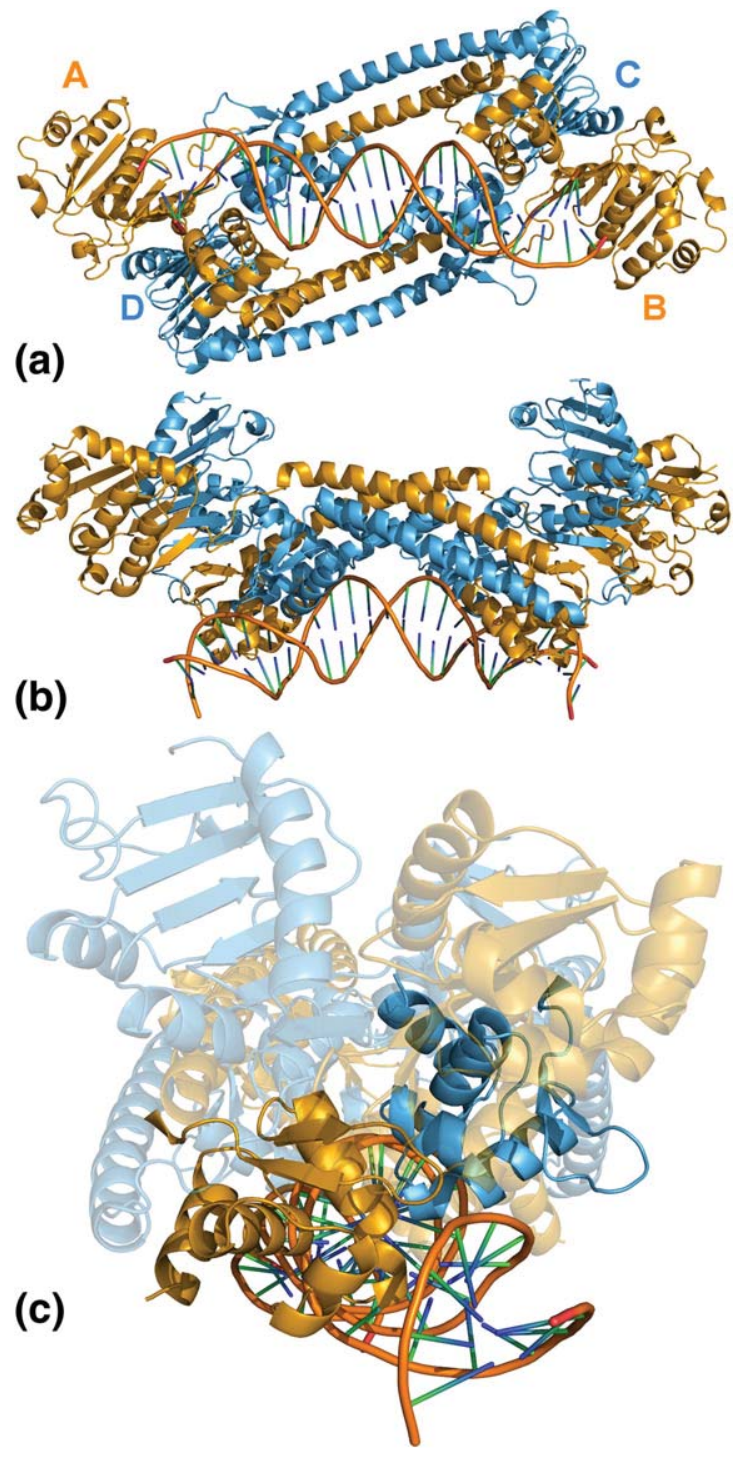

Fig. 1. ecTrmBL2 tetramer in cartoon representation with bound TGM19 DNA. NCS-related chains are shown in the same color. (a) View along the 2-fold symmetry axis. From top to bottom: Chains $\mathrm{C}$ (blue), A (orange), B (orange) and D (blue). $\mathrm{C}$ and $\mathrm{A}$ form one dimer that is NCS related to the other dimer formed by $D$ and $B$. The two ewHTH domains of chains A and B are close to the tetramer ends and are termed "distal" hereafter. The others from chains $C$ and $D$ are termed "proximal" hereafter. (b) View perpendicular to the bent DNA with the 2-fold symmetry axis oriented vertically. The DNA bend angle was calculated to be $63^{\circ}$ [55]. (c) View on one end of the complex along the bent DNA with the 2-fold symmetry axis oriented vertically. This panel is enlarged compared to (a) and (b) and all except the distal ewHTH domain of chain A (orange) and the proximal ewHTH domain of chain $\mathrm{D}$ (blue) is set in a higher transparency to allow a better view of the two ewHTH domains. All structural figures were made with the PyMOL Molecular Graphics System, version 1.7.4.

conformation (Fig. 2d), as well as their symmetry mates (Fig. 1a). The strength of these interactions is reflected by large buried surfaces $\left(1385 \AA^{2}\right.$ and
$1371 \AA^{2}$, respectively) and polar interactions (Fig. 4).

Electrostatic interactions further contribute to the dimer and to the tetramer stability (Fig. 5). The two differently arranged CTDs of chains $C$ and B show opposite surface potentials contributing to the interaction of the two dimers forming the tetramer. Within each dimer, the two ewHTH domains have slightly different surface potentials. In addition, electrostatic interactions also stabilize the dimer as seen from opposite surface potentials of the ewHTH in chain $A$ and the CTD in chain $C$.

\section{TrmBL2-DNA interactions}

When modeling the structure of the TGM19 DNA bound to ecTrmBL2, there was density for 25 bp and the density for the nucleobases was ambiguous at most of the positions prohibiting clear assignment as one of the four bases. Apart from that, the density for the phosphates and the deoxyribose rings were clearly seen. Similarly, in both TGM17 structures, the density indicated $21 \mathrm{bp}$. Moreover, this extra 3-bp density in the case of TGM19 and the 2-bp density in the case of TGM17 at both DNA ends was significantly weaker but because these parts of the model showed up as significant difference map peaks during subsequent cycles of refinement, it was decided to place extra base pairs at these positions and to assume that the DNA is bound to TrmBL2 along the ewHTH domains in various positions. To take this into account, we tried several approaches during the refinement. A grouped occupancy refinement with three copies of TGM19, each positioned along the visible density of the phosphate and ribose backbone with an offset of $3 \mathrm{bp}$ against each other (Fig. 6a), resulted in an increase in $R_{\text {free }}$ by $5 \%$ and was therefore discarded. To reduce the number of refinement parameters, we constructed the parsimonious model shown in Fig. 6b. This model has the known 19-bp segment as its centerpiece but duplicates the first and last $3 \mathrm{bp}$, thus obtaining $25 \mathrm{bp}$. To account for the fact that the total number of base pairs must be 19, we adjusted the occupancy of the first and last three base pairs to one-third, base pairs $4-6$ and 20-22 to two-thirds and base pairs 7-19 to 1.0. We conclude from inspection of the electron density map and the refinement that, in the ecTrmBL2 tetramer, the TGM19 DNA is bent and likely bound at least at three different positions along the four ewHTH domains. Likewise, for the TGM17, there are also three binding positions. This suggests that the four ewHTH domains offer at least three binding sites of roughly the same affinity to the double-stranded TGM DNA that are approximately related to each other by screw axis operations corresponding to a shift of $3 \mathrm{bp}$ in case of TGM19 and of 2 bp in case of TGM17. 


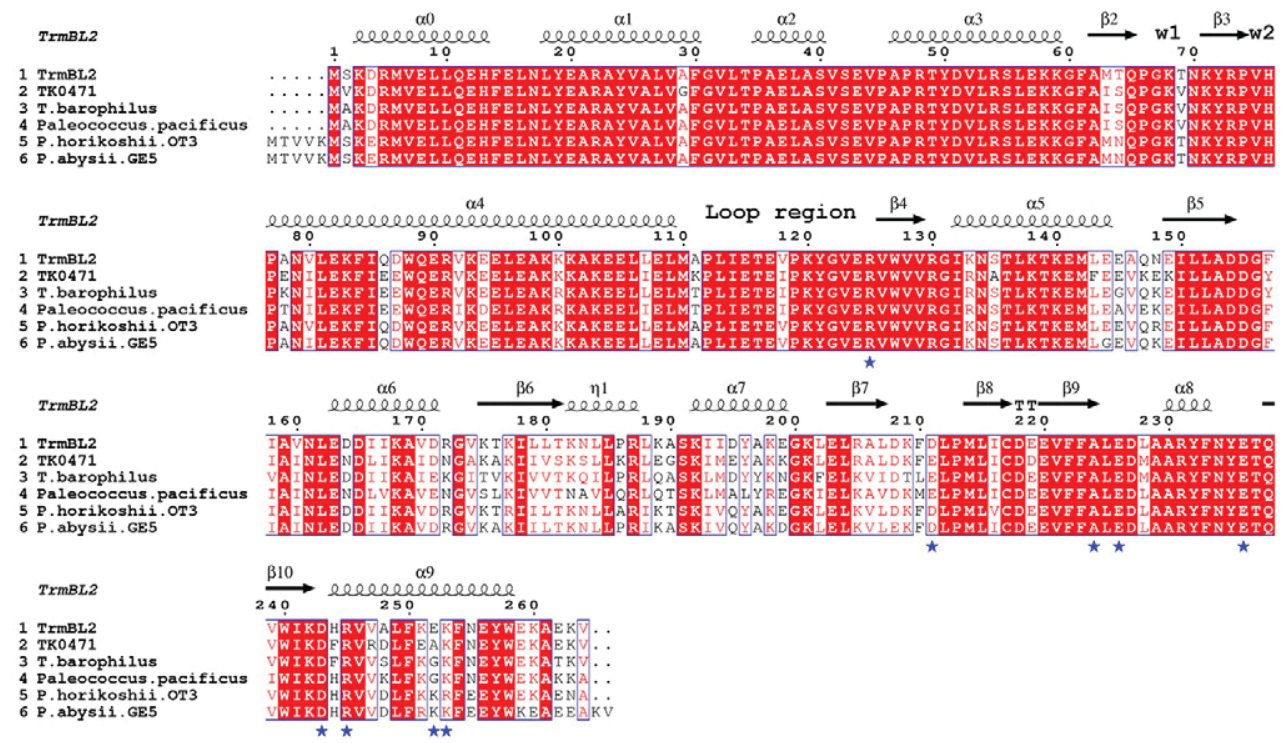

(a)

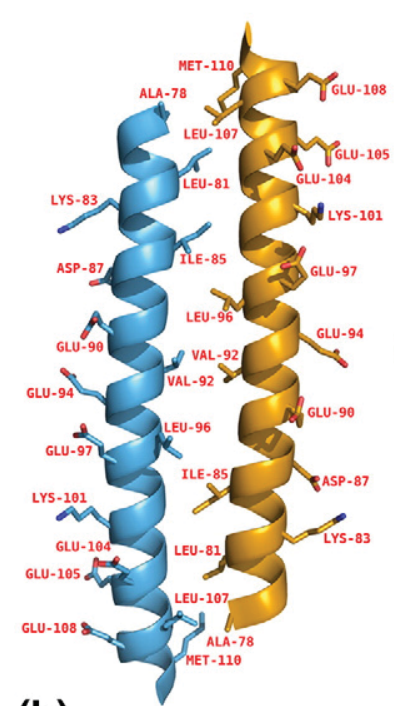

(b)

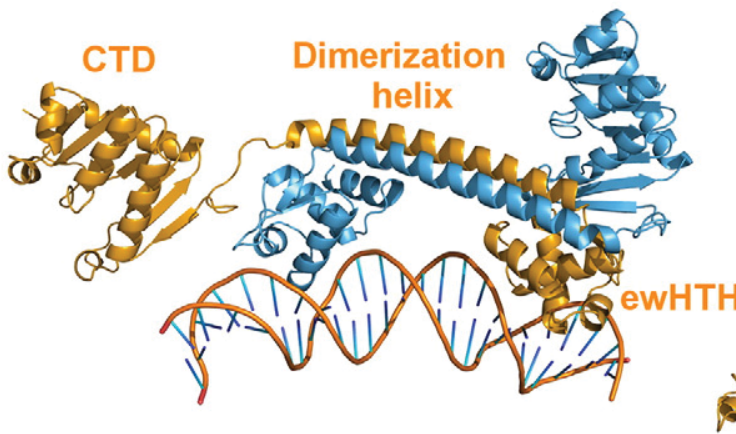

(c)
ewHTHs

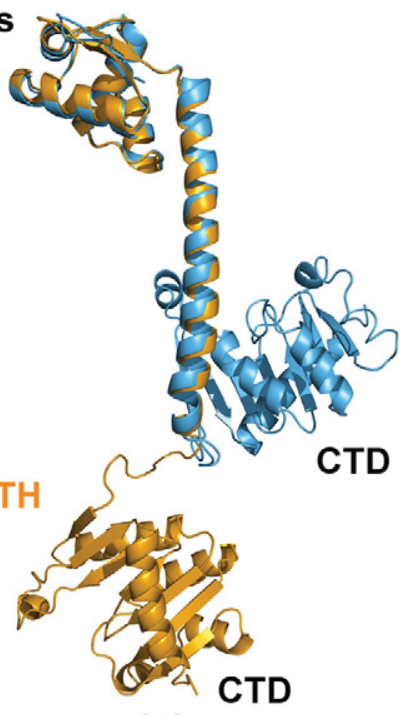

(d)

Fig. 2. (a) Sequence alignment of TrmBL2 homologs in hyperthermophilic archaea labeled with the secondary structure elements from the TrmBL2 structure. The secondary structural elements are defined according to DSSP [56]. The numbering of the secondary structure elements has been altered compared to TrmB [2] to correspond to the conventional labeling of the wHTH proteins. Residues 1-74, 78-109, 112-124 and 125-264 comprise the ewHTH, dimerization helix, loop region and CTD, respectively. The residues forming hydrogen bonds and salt bridges in the interface between the CTDs are labeled with an asterisk. Strictly conserved residues are highlighted in red. Turns, $3_{10}$ helices and wings are denoted as TT, $\eta$ and $w$, respectively. The alignment was generated using the ENDscript server [57]. (b) The coiled coil of two oppositely arranged a4 helices in the TrmBL2 dimer formed by chains $\mathrm{C}$ (blue) and A (orange) in ribbon representation. Solvent-exposed residues and hydrophobic residues lining the interior are labeled. The helices are almost parallel with a crossing angle of $178{ }^{\circ}$ as determined in PyMOL. (c) Broken 2-fold symmetry of the TrmBL2 dimer. View of the TrmBL2 dimer (chain A, orange; chain C, blue) perpendicular to the symmetry axis relating the ewHTH domains and the dimerization helices. For chain $\mathrm{A}$, the domains are labeled. The symmetry is broken due to different conformations of the CTDs. While the distal ewHTH domain of chain A (orange) forms a large buried surface with the CTD of chain $\mathrm{C}$ (blue), this is not the case for the proximal ewHTH of chain $\mathrm{C}$ and the CTD of chain A. (d) Superposition of the two TrmBL2 monomers of the dimer, A in orange and C in blue. The differences in conformation of the two CTDs are obvious. 


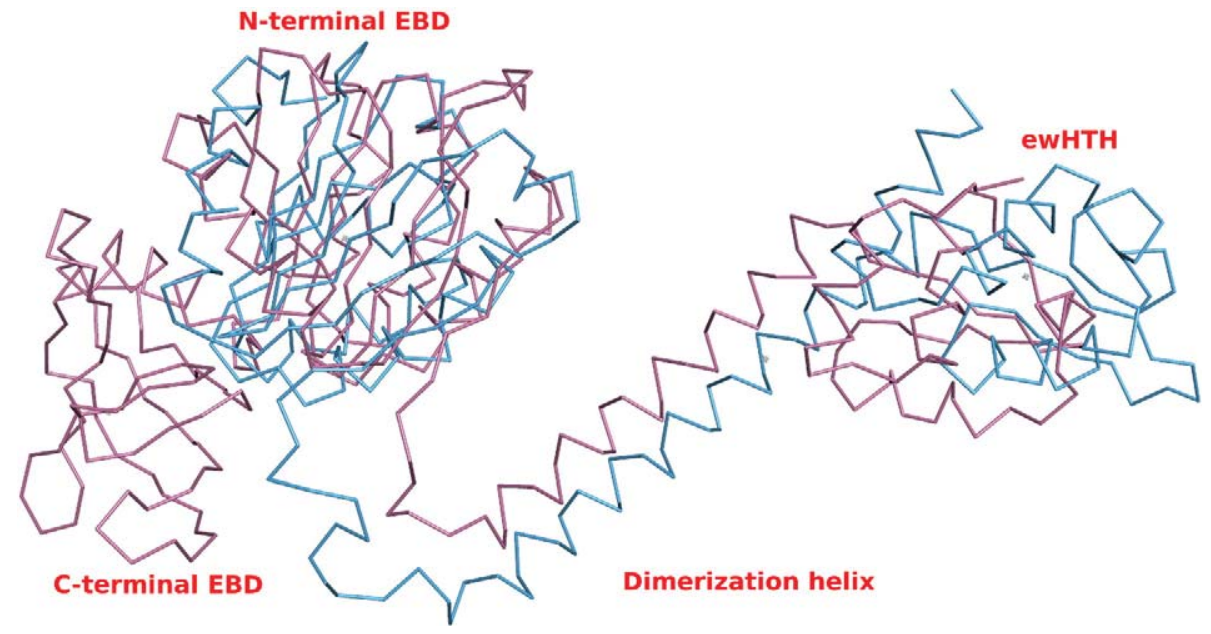

Fig. 3. Superposition of the $C^{\alpha}$ atoms of chain $C$ of TrmBL2 (blue) with those of TrmB (purple). The CTD of this chain of TrmBL2 and the N-terminal subdomain of EBD of TrmB show a similar "trans" arrangement to the ewHTH domain relative to the dimerization helix.

As is the case with most of the nonspecific DNA binding proteins, TrmBL2 interactions with the DNA are mostly confined to the deoxyribose phosphate backbone (Fig. 6c). Moreover, the proximal ewHTH domains of chains $\mathrm{C}$ and $\mathrm{D}$ present more residues for interaction with the DNA backbone than the distal

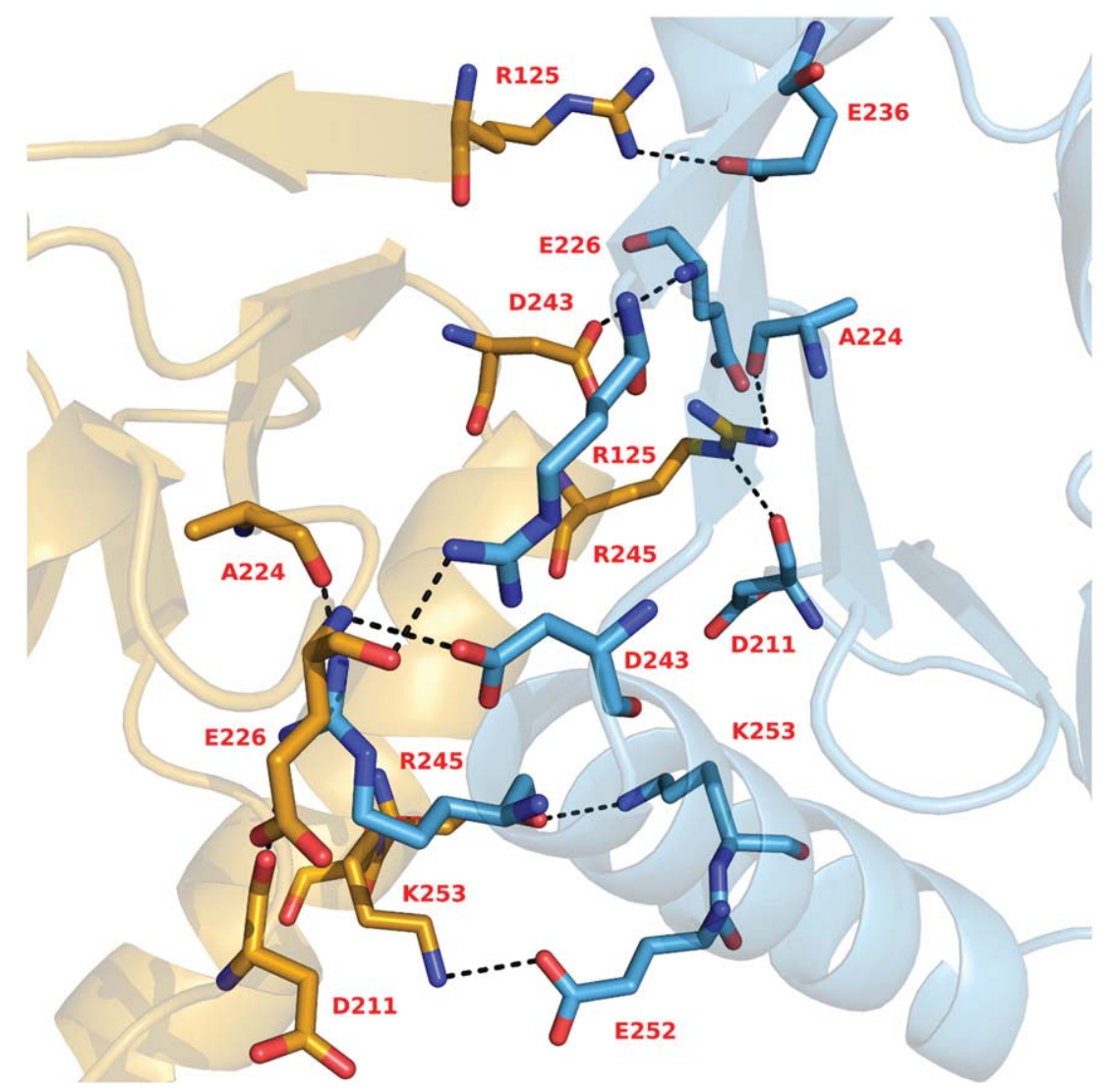

Fig. 4. Interface of the CTDs from chains A (orange) and D (blue) with densely spaced polar interactions. The same interface of CTDs is found in the NCS-related symmetry mate from chains B and C. A separate 2-fold symmetry relates these two interacting CTDs (data not shown). 


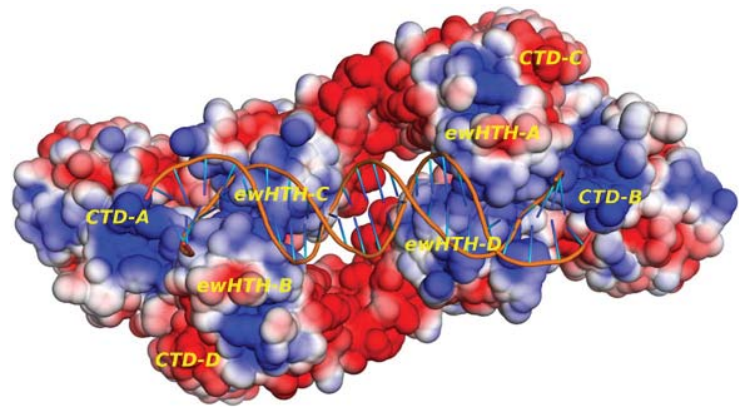

Fig. 5. Surface potential representation of the TrmBL2 tetramer bound to TGM19 DNA. The tetramer is oriented as in Fig. 1, top panel. Electrostatic potential was calculated by APBS [58-60].
ewHTH domains from chains $A$ and $B$. For instance, Arg54 from the proximal ewHTH domain of chain $\mathrm{C}$ and that from its NCS-related chain D form hydrogen bonds with the DNA backbone in case of the ecTrmBL2-TGM19 complex whereas the corresponding Arg54 residues from the distal ewHTH of chain $A$ and its NCS-related chain B point away from the DNA backbone. In accord with this asymmetry, buried surface area calculations [19] also show that the recognition helix $\alpha 3$ from the proximal ewHTH of chain $\mathrm{C}$ buries a larger surface with DNA $\left(441 \AA^{2}\right)$ than the corresponding helix in the distal ewHTH of chain A $\left(238 \AA^{2}\right)$. Because of the 2-fold NCS that relates the two dimers, the same is true for the BD dimer. The bending of the DNA at the ends could explain such an anomaly.

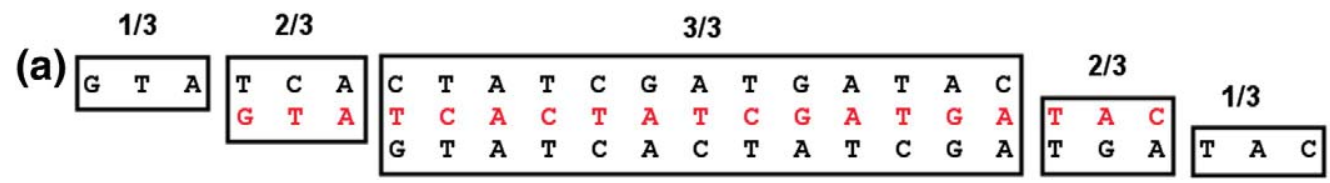

$\begin{array}{llllllllllllllllllllllllllllll}\text { (b) } & G & T & A & G & T & A & T & C & A & C & T & A & T & C & G & A & T & G & A & T & A & C & T & A & C\end{array}$

(c)
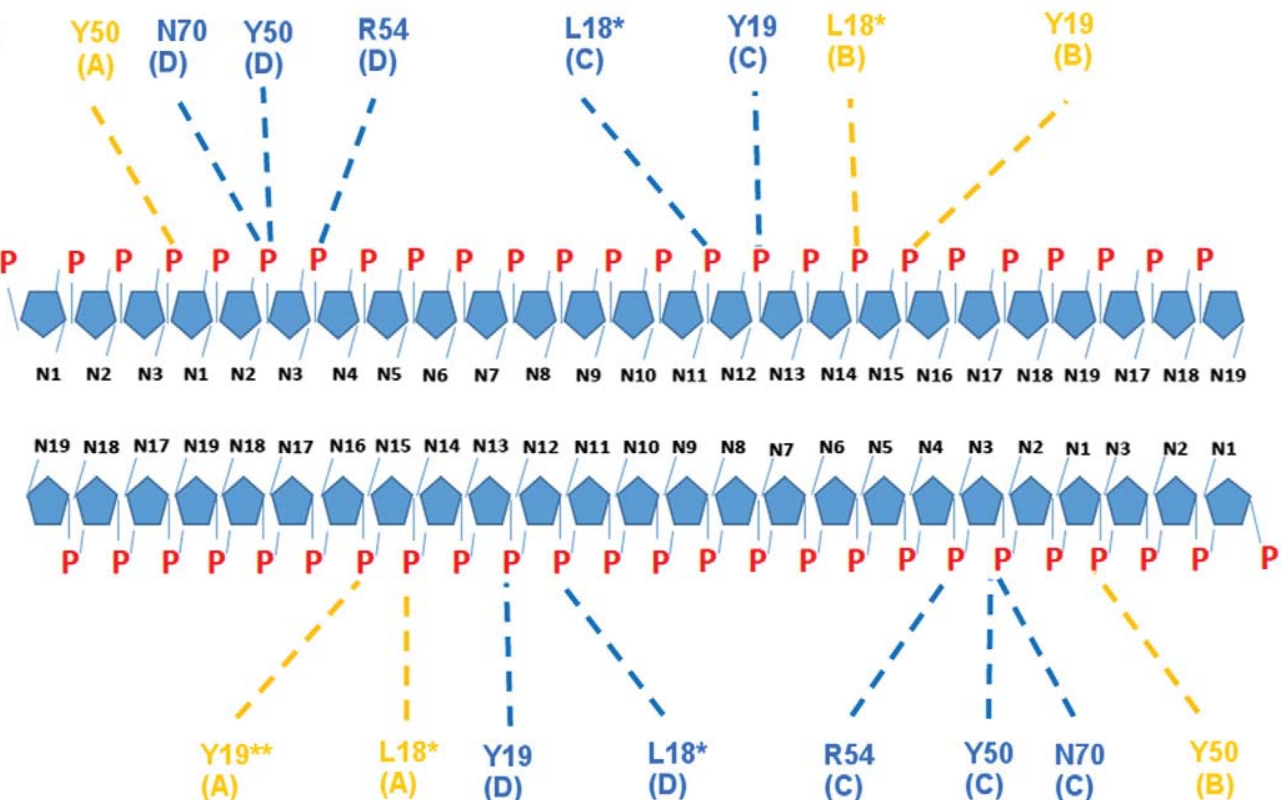

Fig. 6. Occupancy refinement and TrmBL2-TGM19 interactions. The TGM sequence is shown in red. Amino acids interacting with the sugar-phosphate backbone are shown in the same color as the chains they belong to (Fig. 1), which are indicated below each residue. (a) Occupancy refinement with three copies of TGM19. The sequences above and below the TGM sequence (colored red) are shifted by $3 \mathrm{bp}$ at the $5^{\prime}$ and $3^{\prime}$ ends, respectively. The occupancies of the base pairs are shown on top. Grouped refinement with three copies of TGM19 sequence resulted in an increase in $R_{\text {free. }}$ (b) The 25-bp sequence that was fitted in the density. The TGM19 sequence (red) is shown in the middle with the 3-bp extensions at the $5^{\prime}$ and $3^{\prime}$ ends shown in black. Refinement got better by placing only one nucleotide at each position with adjusted occupancy along the 25 -bp density stretch. The occupancy of the residues is adjusted so that the total number of base pairs adds to 19. It is important to note that two base pairs with equal probability occupy positions 4-6 and 20-22, and three base pairs with equal probability occupy positions 7-19. (c) Symmetrical interactions of the amino acids from the four ewHTH domains with the sugar-phosphate backbone of TGM19. Residues interacting with the DNA backbone within $3.5 \AA$ are shown. *Main chain contacts with the TGM19 sugar-phosphate backbone. ${ }^{* *} 3.67-\AA ̊$ distance from the TGM19 sugar-phosphate backbone. 
The majority of the helix-turn-helix proteins that bind DNA in the major groove form sequence-specific contacts with the dsDNA by polar interactions of the residues from the recognition helix $\alpha 3$ with nucleobases. In case of TrmBL2, only Arg48 residues from chains $A$ and $B$ approach the nucleobases within $3.5 \AA$ atoms for potential hydrogen bonding interactions. The only other residues that approach the nucleobases within $3.5 \AA$ are the apolar Pro47 residues from all four ewHTH domains (Fig. 7, top panel).

\section{The DNA-free structure of pfTrmBL2}

The DNA-free form of pfTrmBL2 could be solved by molecular replacement using ecTrmBL2 as the search model (see Table 1) and revealed a tetramer in the asymmetric unit. Superposition of

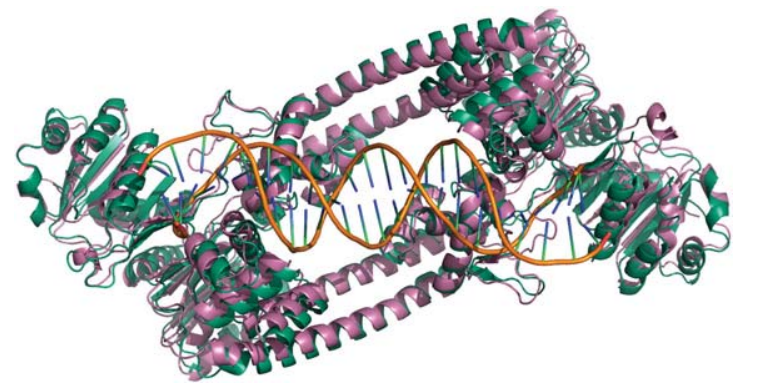

Fig. 8. Superposition of the ecTrmBL2-TGM19 complex (magenta) and DNA-free pfTrmBL2 (green).

both structures was possible with an RMSD of $2.8 \AA$ (calculated by SSM superpose in Coot) (Fig. 8) and shows only subtle changes upon DNA binding.
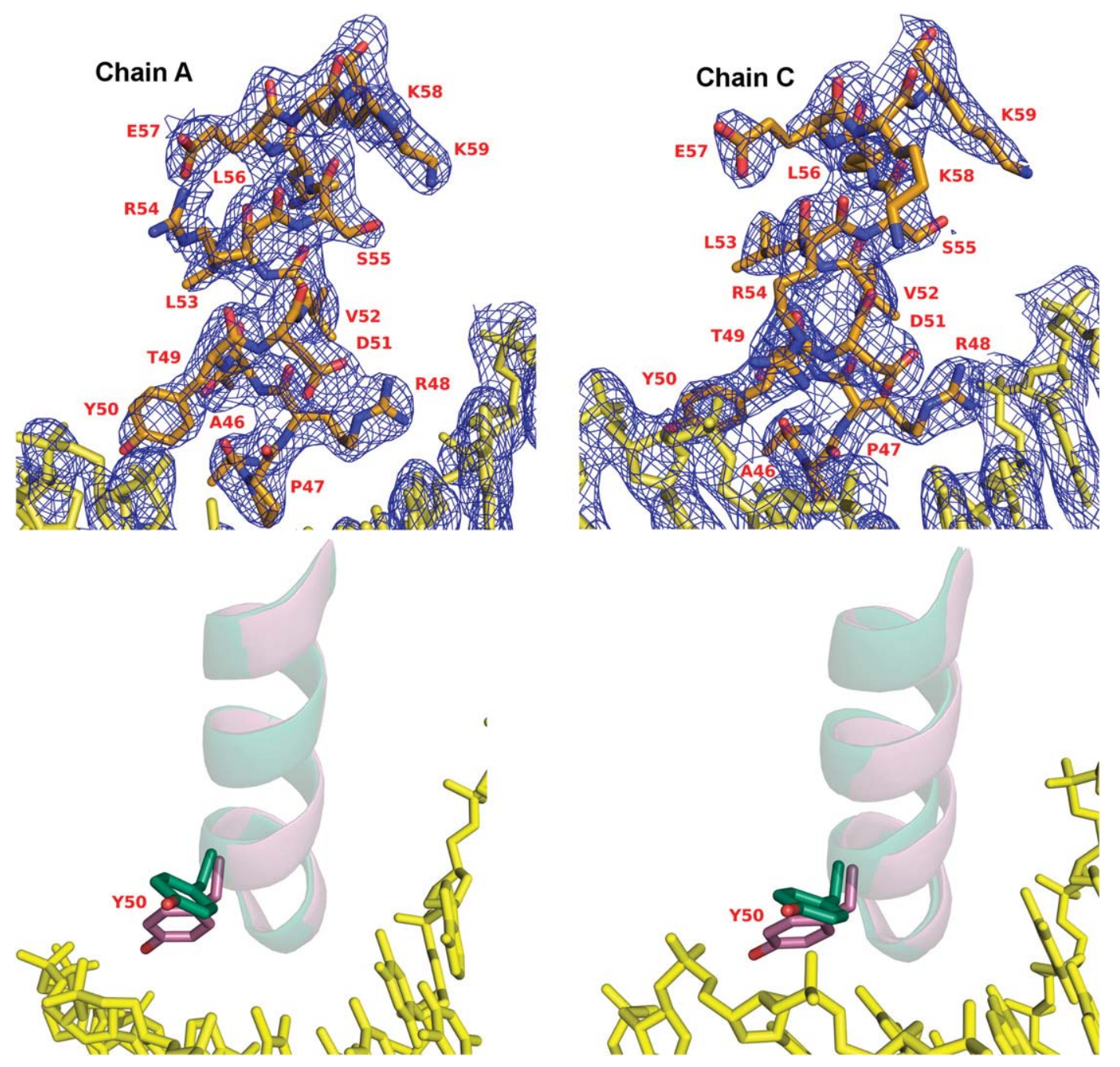

Fig. 7. Top panel: details of the interaction of the recognition helices with TGM19 in the AC dimer. The $2 F_{\mathrm{o}} F_{\mathrm{c}}$ electron density map contoured at $1 \sigma$ is shown with the amino acids in stick representation. DNA is shown in stick representation (yellow). The ewHTH of chain $\mathrm{A}$ is distal, and that of chain $\mathrm{C}$ is proximal. Bottom panel: different side chain conformations of Tyr50 in the DNA-free pfTrmBL2 structure (green) and the ecTrmBL2-TGM19 DNA-bound form (magenta) in the AC dimer. 
A zoom-in into the DNA binding residues of the ewHTH domains shows that the side chain of Tyr50 from all four ewHTH domains in the ecTrmBL2-TGM19 complex swings toward the DNA backbone compared to the DNA-free pfTrmBL2 (Fig. 7, bottom panels).

\section{Discussion}

\section{TrmBL2 was discovered together with specific repressor molecules}

TrmBL2 was originally assumed to act as a repressor akin to TrmB and TrmBL1 that control expression of transport systems and carbohydratemetabolizing enzymes in $P$. furiosus [3]. This prompted us to crystallize the protein in complex with the corresponding dsDNA TGM operator sequence. Among these TrmB homologs, TrmBL2 is unusual for three reasons: it lacked an inducer binding site, it was conserved in all Thermococcales and it showed a surprisingly high degree of sequence identity among the different Thermococcales (Fig. 2a). Moreover, it is maintained at a high level of $37 \mu \mathrm{M}$ in T. kodakarensis cells [9]. Band shift experiments revealed that it binds to the TGM sequence with low affinity but not exclusively [3]. For the highly homologous TK0471 protein from $T$. kodakarensis [8,9], it was shown to bind nonspecifically to DNA and impart on it the appearance of a thick, stiff fiber.

\section{The asymmetry of the dimeric structure allows for tetramerization}

The structure of TrmBL2 shares the overall domain organization and dimerization mode with TrmB except that it lacks the C-terminal sugar-binding domain. Apart from these general similarities, the TrmBL2 dimer has a distinctly asymmetric conformation of the CTDs that is absent in TrmB and other dimeric repressor proteins. The 13-residue loop that connects the dimerization helix to the C-terminal domain allows for this flexibility.

The observation that pfTrmBL2 forms tetramers in solution suggests that the tetramer might be the native oligomeric form in the absence of DNA although ecTrmBL2 forms dimers.

The two dimers of the tetramer bind to DNA with an offset of approximately $2 \mathrm{bp}$ and cover almost half of the DNA circumference. This staggered arrangement of the dimers on the DNA ensures a larger coverage of the DNA surface that, along with its nonspecific binding mode, could hinder access to various DNA modifying enzymes [8].

Although structures of a large number of dimeric bacterial repressors are available, the same is not the case for complete tetrameric assemblies. TtgV represents one bacterial repressor whose complete tetrameric structure, alone and in complex with DNA, has been solved [20]. In TtgV, a large conformational change of the N-terminal domains occurs upon DNA binding. In contrast, TrmBL2 has very similar DNAbound and DNA-free structures. The way the four $\mathrm{N}$-terminal DNA binding domains are arranged on the DNA is also different between TtgV and TrmBL2. Whereas in $\mathrm{Ttg} V$, the $\mathrm{N}$-terminal domains bind only to one face of the DNA, in TrmBL2, owing to their staggered arrangement; they make a larger fraction of the DNA inaccessible to macromolecules. On the other hand, the 19-bp dsDNA in the TrmBL2 complex is more accessible to small solvent molecules than the 42-bp dsDNA in the TtgV complex as in both complexes calculations [21] show that $20 \%$ of the DNA is inaccessible to water. This is quite in agreement with the fact that $T \operatorname{tg} V$ is a specific binder and therefore contacts the DNA more intimately than TrmBL2, which is a nonspecific binder and forms very few contacts with the DNA bases.

\section{Structural homologs of the TrmBL2 CTD}

A search for structural homologs using PDBeFold [13] showed that the C-terminal domain of TrmBL2 bears structural homology to the phospholipase $\mathrm{D}$ domain [22]. The $\mathrm{HxK}(\mathrm{x})_{4}$ D motif (HKD signature motif) found in proteins that have phospholipase activity is absent in TrmBL2. The phospholipase D-like domains are also found in endonucleases, toxins and as structural components of pox virus envelope [23,24]. In case of TrmBL2, the C-terminal domain seems to function as a tetramerization domain and could in principle also act as a scaffold for protein-protein interactions [8].

\section{The coiled-coil domain}

The coiled-coil domain as a dimerization motif is a common feature found in many bacterial and eukaryotic transcription factors. In the case of TrmB, TrmBL2, $\mathrm{BmrR}$ and proteins of similar dimeric structure, it places the DNA binding domains in a manner that allows for their simultaneous interactions with adjacent major grooves. The dimerization helix a4 in TrmBL2 is longer than in TrmB but of similar length as in Sso10a [25], and its hydrophobic interface promotes the dimer formation. In TrmBL2, the two a-helices are almost parallel ( $178^{\circ}$ angle as calculated in PyMOL). A similar orientation of the dimerization helices is also found in $\operatorname{TrmB}\left(175^{\circ}\right)$ [2]. This contrasts with other dimeric DNA binding proteins of known function like BmrR [26] $\left(161^{\circ}\right)$, Sso10a [25] $\left(157^{\circ}\right)$ and CAP [27] $\left(156^{\circ}\right)$.

\section{The N-terminal ewHTH domain}

A search for similar structures using PDBeFold revealed that TrmBL2 is a member of the winged 
helix-turn-helix (wHTH) family of DNA binding proteins that are widely distributed across all the three domains of life and are involved in almost every aspect of nucleic acid metabolism [28]. Within the wHTH family, TrmBL2 falls under the ewHTH subfamily that is characterized by extra helices, $\alpha 0$ at the $\mathrm{N}$-terminus and $\alpha 4$ at the $\mathrm{C}$-terminus of the canonical wHTH domain [29]. The top structural hits based on the PDBeFold Q-score included PKZ Zalpha (PDB ID 4LB5; a zebrafish Z-DNA-dependent protein kinase PKZ), meiotic recombination protein HOP2 (PDB ID 2MH2) and C-terminal domain of hRPA32 (PDB ID 1Z1D), all from eukaryotic organisms.

\section{Surface electrostatics of the wHTH domains bound to DNA}

Most of the wHTH proteins carry a strong electropositive surface on the face that is involved in DNA binding. In a majority of cases, the strongly electropositive recognition helix a3 is involved in base-specific contacts. In cases where other structural features are employed for nucleobase recognition in the major groove, like wing 1 in case of $\mathrm{hFRX} 1$, the wing 1 is more electropositive than the recognition helix $[30,31]$. A comparison of the electrostatic surface of the wHTH domains from TrmBL2 with other members of wHTH proteins whose DNA-bound structures are available (Fig. 9) reveals that the DNA-facing surface of TrmBL2 is significantly less electropositive.

As mentioned earlier, in the TrmBL2-DNA complex structure, the majority of the interactions are with the DNA backbone and only Pro47 from all four ewHTH domains and Arg48 from the ewHTH domains of chains $A$ and $B$ make contacts with the DNA bases. This lack of polar residues in the close vicinity of the nucleobases is reflected in the low polarity of the surface potential (Fig. 9f).

\section{Functional and structural similarities with bacterial H-NS}

In an attempt to describe the concurrent role of transcriptional repression and chromosome shaping, it has been suggested that TrmBL2 functions in a similar manner as bacterial H-NS by forming stiff nucleoprotein filaments [32,33]. A structural comparison of the two reveals that $\mathrm{H}$-NS also contains the C-terminal wHTH DNA binding domain [34] and an $\mathrm{N}$-terminal domain that has been proposed to facilitate oligomerization [35]. Since the $\mathrm{N}$ - and $\mathrm{C}$-terminal structures of $\mathrm{H}$-NS have been solved separately, the orientation of the wHTH domain with respect to the $\mathrm{C}$-terminal oligomerization domain is not known. However, the orientation of the secondary structural elements in bacterial H-NS wHTH domain is reversed as compared to TrmBL2 and other wHTH proteins so that a similar function would be conceivable only by convergent evolution.

\section{Relation of TrmBL2 with other nonspecific archaeal DNA binding proteins}

As already mentioned, recent work has accumulated evidence for nonspecific DNA binding of TrmBL2 and its close homologs. The TrmBL2 structure supports this view. The low polarity and the subtle asymmetry of the distal and the proximal ewHTH-DNA interfaces of the dimer fit to a protein design that avoids specific polar contacts with nucleobases. The blurred electron density map of the nucleobases could be explained by a superposition of three TGM motifs approximately related to each other by screw axis symmetry operations shifting the dsDNA by $3 \mathrm{bp}$. TrmBL2 is composed of two dimers, each of which is structurally related to the specific repressor proteins of the TrmB family that bind a specific operator sequence by placing a pair of ewHTH domains in close association with two adjacent major grooves. It is therefore not surprising that, in TrmBL2, such a dimer of dimers designed for nonspecific binding will use mismatching of four ewHTH binding sites and apolar interaction surfaces to offer several low-affinity binding sites to DNA.

This design of the DNA binding surface is reminiscent of archaeal chromatin proteins Sac7d and Sso7d from the Sul7d family. The crystal structure of Sso7d in complex with DNA shows that this protein presents an apolar surface to DNA [36]. Thriving at very high temperatures means that the DNA of extremophiles is susceptible to a host of insults that include thermal denaturation and radiolysis [37]. Archaea have evolved a host of chromatin binding proteins such as $\mathrm{HTa}, \mathrm{MC} 1$, Sul7d family of proteins, Cren7 and Alba that stabilize the DNA in order to counter the effect of extreme temperatures on DNA structure. All of these proteins show very low nucleotide sequence specificity and stabilize the DNA at extreme temperatures against denaturation [38-43], a role that might also hold true for TrmBL2.

TrmBL2 with its ewHTH domains that are infrequent in the archaeal architectural proteins obviously has evolutionary roots in the specific repressor protein families of bacteria and archaea. The evolution of an apolar interaction surface with DNA that TrmBL2 shares with Sso7d could be a case of convergent evolution.

\section{Materials and Methods}

His $_{6}$ purification tag introduction into $P$. furiosus TrmBL2 (pfTrmBL2)

The $\mathrm{His}_{6}$ tag was introduced at the $\mathrm{N}$-terminus by double cross-over of homologous sequences upstream and downstream of TrmBL2. For the construction of the corresponding DNA, four different DNA fragments were combined step by step by single overlap extension 


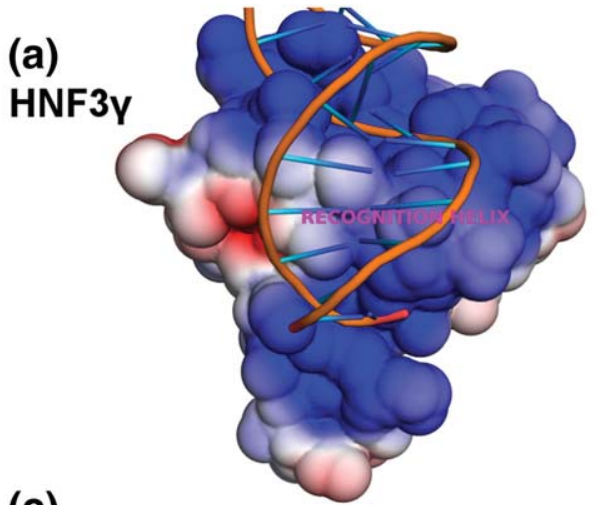

(c)

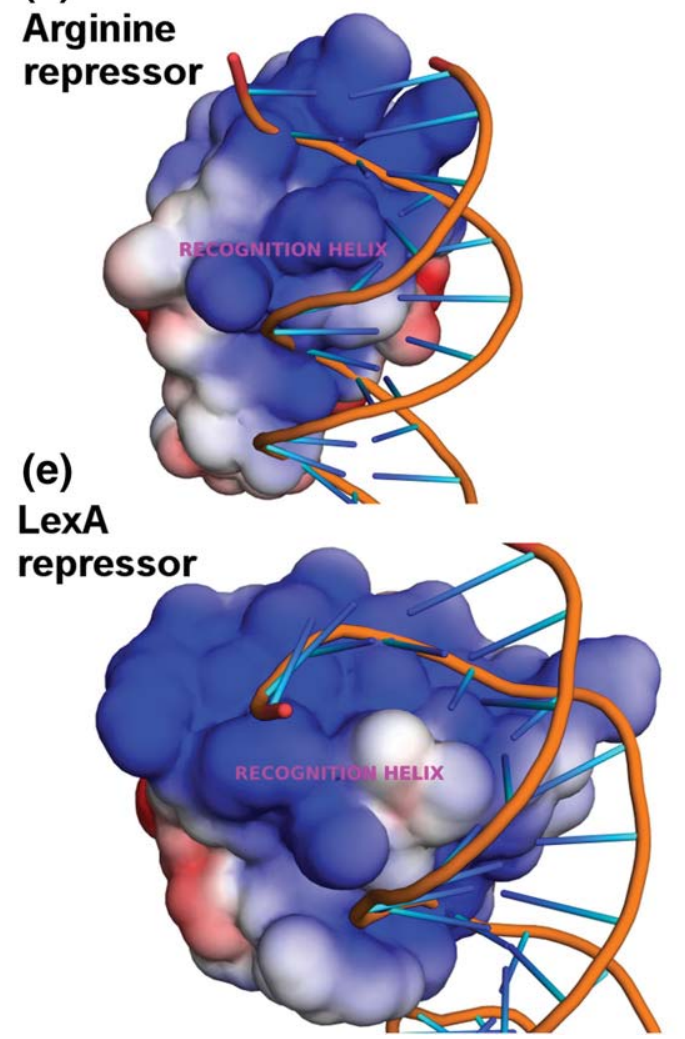

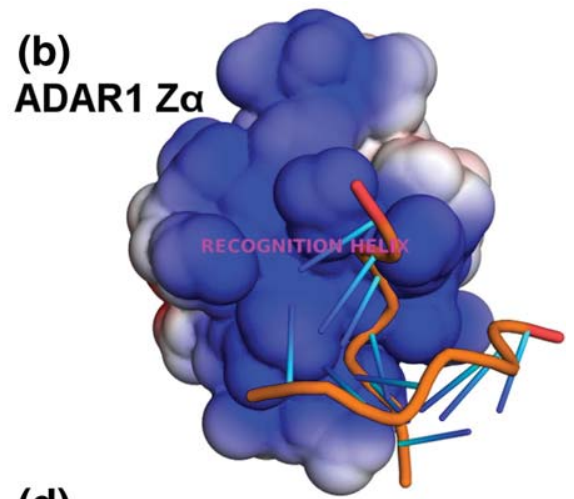

(d)

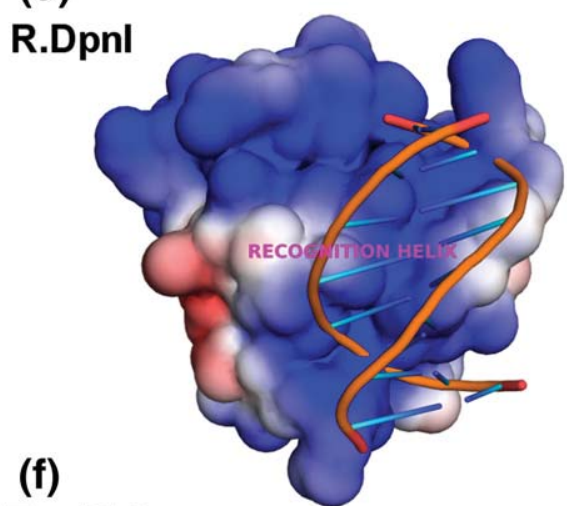

TrmBL2

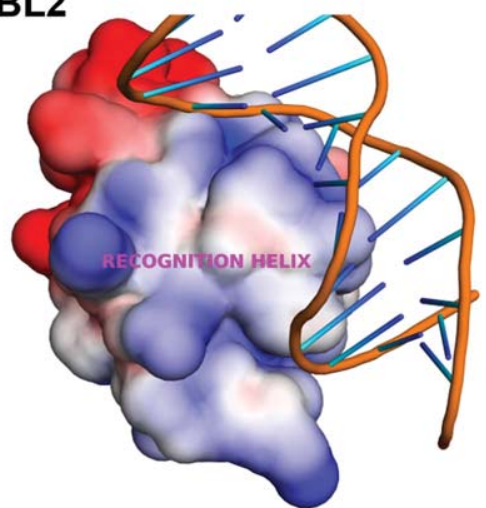

Fig. 9. Comparison of the surface electrostatic potential of wHTH domains in typical bacterial repressors with TrmBL2 (distal ewHTH from chain A). While the former face DNA with predominantly positive surface potential (blue), the latter is distinctly apolar. The potential was calculated with APBS [58-60]. The PDB codes are (a) 1VTN, (b) 1QBJ, (c) 2P5L, (d) 4ESJ and (e) 3JSP.

PCR reactions. The primers are shown in Supplementary Table 1. The complete DNA fragment was transformed into $P$. furiosus as described previously $[44,45]$. The transformants were selected with the antibiotics simvastatin and pure cultures were isolated after plating the cells on solidified medium in the presence of $10 \mu \mathrm{M}$ simvastatin. The integration of the DNA fragment was verified by analyzing corresponding PCR products. The resulting strain MURPf10 with the His $_{6}$-tagged TrmBL2 was grown in a 100-I fermenter to produce cell mass for large-scale purification of the protein [44].

\section{Cloning and expression of TrmBL2 in E. coli BL21 DE3 (ecTrmBL2)}

A synthetic, codon-optimized gene construct of $P$. furiosus TrmBL2 in kanamycin-resistant pET24d vector was ordered from Trenzyme GmbH (Konstanz, Germany). The construct was transformed into $E$. coli BL21 DE3 expression strain and cells were grown in Kanamycin supplemented LB medium at $37^{\circ} \mathrm{C}$. Protein expression was induced by adding $1 \mathrm{mM}$ IPTG to the cell culture at $\mathrm{OD}_{600}$ of 0.6 . The culture was further grown for $4 \mathrm{~h}$ postinduction. Cells were harvested and stored at $-80^{\circ} \mathrm{C}$. 


\section{Purification of pfTrmBL2 and ecTrmBL2}

For the purification of pfTrmBL2, 10-g cells were resuspended in $50 \mathrm{ml}$ lysis buffer containing $40 \mathrm{mM}$ Hepes ( $\mathrm{pH} 7.5$ ), $1 \mathrm{M} \mathrm{NaCl}, 20 \mathrm{mM}$ imidazole, $20 \%$ glycerol, one-third protease inhibitor tablet (Roche) and $10 \mu \mathrm{g} / \mathrm{ml}$ of DNase (Roche) and were incubated at room temperature for $30 \mathrm{~min}$. The cell lysis was carried out by a cell disrupter (Constant Systems Limited, UK) and the lysate was centrifuged at $185,500 \mathrm{~g}$ for $60 \mathrm{~min}$. The supernatant was filtered and loaded onto a $5-\mathrm{ml} \mathrm{Ni}$ HisTrap FF column (GE Healthcare) equilibrated with Buffer A [40 mM Hepes (pH 7.5), 20 mM imidazole, $1 \mathrm{M}$ $\mathrm{NaCl}$ and $20 \%$ glycerol]. The protein was eluted by a linear imidazole gradient with Buffer B [40 mM Hepes (pH 7.5), $0.5 \mathrm{M}$ imidazole, $1 \mathrm{M} \mathrm{NaCl}$ and $20 \%$ glycerol] on AKTA purifier. pfTrmBL2-containing fractions as analyzed by SDS-PAGE were pooled, concentrated and loaded onto a 60-ml Superdex 200 column preequilibrated with $40 \mathrm{mM}$ Hepes ( $\mathrm{pH} 7.5), 150 \mathrm{mM} \mathrm{NaCl}$ and $20 \%$ glycerol.

For ecTrmBL2 purification, 4-g cells (wet weight) were resuspended in lysis buffer consisting of $40 \mathrm{mM}$ Hepes (pH 7.5), $150 \mathrm{mM} \mathrm{NaCl}$ and $20 \%$ glycerol and lysed using the French press at 16,000 psi. The lysate was heated at $80{ }^{\circ} \mathrm{C}$ for $25 \mathrm{~min}$ and later centrifuged at $185,500 \mathrm{~g}$ for $60 \mathrm{~min}$. The supernatant was diluted to a final $\mathrm{NaCl}$ concentration of $50 \mathrm{mM}$, filtered and loaded onto a Q-Sepharose anion-exchange column equilibrated with Buffer A [40 mM Hepes ( $\mathrm{pH} 7.5)$ and $150 \mathrm{mM}$ $\mathrm{NaCl}$. Protein elution was carried out by a linear gradient with Buffer B [40 mM Hepes ( $\mathrm{pH} 7.5)$ and $1 \mathrm{M} \mathrm{NaCl}$ ] on AKTA purifier. Fractions containing ecTrmBL2 as analyzed by SDS-PAGE were pooled, concentrated and loaded onto a $60-\mathrm{ml}$ Superdex 200 column preequilibrated with buffer containing $40 \mathrm{mM}$ Hepes $(\mathrm{pH} 7.5)$ and $150 \mathrm{mM} \mathrm{NaCl}$.

\section{Selenomethionine incorporation into ecTrmBL2}

For selenomethionine incorporation, cells were grown overnight in $\mathrm{M} 9$ minimal medium supplied with $20 \mathrm{ml}$ of $20 \%$ glucose, $2 \mathrm{ml}$ of $1 \mathrm{M} \mathrm{MgSO}_{4}$ and $100 \mu \mathrm{l}$ of $1 \mathrm{M} \mathrm{CaCl}_{2}$ per liter (hereafter called M9 PLUS) and the overnight culture was used to inoculate fresh M9 PLUS medium. To inhibit methionine synthesis in E. coli, an amino acid mixture consisting of $100 \mathrm{mg}$ lysine, phenylalanine and threonine; $50 \mathrm{mg}$ of isoleucine, leucine and valine; and $60 \mathrm{mg}$ of selenomethionine was added to 11 of the cell culture at $O D_{600}$ of 0.5 . Cells were further grown for $15 \mathrm{~min}$ before being induced by $1 \mathrm{mM}$ IPTG. Cells were harvested after an overnight growth at $18^{\circ} \mathrm{C}$. The purification protocol was the same as that for the native ecTrmBL2.

\section{Crystallization}

For crystallization of pfTrmBL2, the protein was concentrated to $16 \mathrm{mg} / \mathrm{ml}$. For ecTrmBL2 crystallization, the protein was concentrated to $10 \mathrm{mg} / \mathrm{ml}$ and mixed with either 19-bp TGM (5' GTATCACTATCGATGATAC $3^{\prime}$ ) hereafter referred to as TGM19 or 17-bp TGM sequence (5' TATCACTATCGATGATA $3^{\prime}$ ) hereafter referred to as TGM17, in a 1:3.4 molar ratio. The HPLC purified nucleotides were purchased from Thermo Fisher Scientific (Ulm, Germany). The mixture was incubated at $37^{\circ} \mathrm{C}$ for $30 \mathrm{~min}$. Screens were set up in 96-well plates using the Phoenix ARI crystallization robot. Initial crystallization hits were fine-screened and final crystals of pfTrmBL2 were obtained in $0.2 \mathrm{M}$ calcium acetate and $21 \%$ polyethylene glycol 3350. ecTrmBL2 crystals were obtained in $60 \%$ methyl-2,4-pentanediol and $0.1 \mathrm{M}$ Hepes $(\mathrm{pH} 6.5)$ for 19-mer complex and in $0.1 \mathrm{M}$ imidazole $\mathrm{HCl}(\mathrm{pH} 8.0)$, $30 \%$ methyl-2,4-pentanediol and $10 \%$ polyethylene glycol 4000 for the 17 -mer complexes.

\section{Data collection, phasing, structure determination and refinement}

Crystals were flash-cooled in liquid $\mathrm{N}_{2}$, and data were collected at beamline X06SA or X06DA of Swiss Light Source, Villigen, Switzerland. Data were processed by XDS $[46,47]$, and for the selenomethionine TrmBL2-TGM17 complex, anomalous scatterers were found from a single data set (Se-SAD) by the HySS submodule [48] of the Phenix package [49]. A partial model was built by AutoBuild (Phenix) and further model building was performed in Coot [50]. Refinement was carried out in Phenix with chain $A$ being NCS restrained to chain B and with chain C being NCS restrained to chain $D$. For all four models, paired refinement [47] was performed in steps of $0.1 \AA$ to determine the high-resolution cutoff beyond which additional data did not improve the model.

TLS groups used in refinement were selected from the output of TLS motion determination server [51]. Subsequently, structures of TrmBL2-TGM19 and TrmBL2-TGM17 complexes were solved by molecular replacement (Phaser [52]).

The apo-TrmBL2 structure was solved by molecular replacement by using the TrmBL2-TGM19 structure as the molecular replacement model (TFZ $=30.3, \mathrm{LLG}=5989)$. Initial model refinement was aided by Rosetta (Phenix) [53] followed by refinement in Phenix. See Table 1 for details. Buried surfaces were calculated using the EPPIC Web server [54].

\section{PDB depositions}

The structures have been deposited in the PDB under accession numbers 5BOX, 5BPD, 5BPI and 5BQT.

Supplementary data to this article can be found online at http://dx.doi.org/10.1016/j.jmb.2015.08.012.

\section{Acknowledgements}

We are grateful to the staff of Swiss Light Source, particularly to the help of Dr. Meitian Wang. With Dr. Karsten Schäfer, we had many useful discussions. M.U.D.A. acknowledges the support of Konstanz Research School Chemical Biology. This work was partially funded by the Deutsche Forschungsgemeinschaft grants WE 962/12-1 and BO 545/19-1. 
The research leading to these results has received funding from the European Community's Seventh Framework Programme (FP7/2007-2013) under BioStruct-X (grant agreement $\left.\mathrm{N}^{\circ} 283570\right)$.

\section{Keywords:} TrmBL2;

nonspecific DNA binding; chromatin protein; protein-DNA complex; Archaea

Abbreviations used:

ewHTH, extended winged helix-turn-helix; wHTH, winged helix-turn-helix; dsDNA, double-stranded DNA; NCS, noncrystallographic symmetry; HTH, helix-turnhelix.

\section{References}

[1] S.J. Lee, C. Moulakakis, S.M. Koning, W. Hausner, M. Thomm, W. Boos, TrmB, a sugar sensing regulator of $A B C$ transporter genes in Pyrococcus furiosus exhibits dual promoter specificity and is controlled by different inducers, Mol. Microbiol. 57 (2005) 17971807.

[2] M. Krug, S.J. Lee, W. Boos, K. Diederichs, W. Welte, The threedimensional structure of TrmB, a transcriptional regulator of dual function in the hyperthermophilic archaeon Pyrococcus furiosus in complex with sucrose, Protein Sci. 22 (2013) 800808.

[3] S.J. Lee, M. Surma, S. Seitz, W. Hausner, M. Thomm, W. Boos, Characterization of the TrmB-like protein, PF0124, a TGM-recognizing global transcriptional regulator of the hyperthermophilic archaeon Pyrococcus furiosus, Mol. Microbiol. 65 (2007) 305318.

[4] S.-J. Lee, A. Engelmann, R. Horlacher, Q. Qu, G. Vierke, C. Hebbeln, M. Thomm, W. Boos, TrmB, a sugar-specific transcriptional regulator of the trehalose/maltose $A B C$ transporter from the hyperthermophilic archaeon Thermococcus litoralis, J. Biol. Chem. 278 (2003) 983990.

[5] A. Gindner, W. Hausner, M. Thomm, The TrmB family: A versatile group of transcriptional regulators in Archaea, Extremophiles 18 (2014) 925936.

[6] T. Kanai, J. Akerboom, S. Takedomi, H.J. van de Werken, F. Blombach, J. van der Oost, T. Murakami, H. Atomi, T. Imanaka, A global transcriptional regulator in Thermococcus kodakaraensis controls the expression levels of both glycolytic and gluconeogenic enzyme-encoding genes, J. Biol. Chem. 282 (2007) 3365933670.

[7] H.J. van de Werken, C.H. Verhees, J. Akerboom, W.M. de Vos, J. van der Oost, Identification of a glycolytic regulon in the archaea Pyrococcus and Thermococcus, FEMS Microbiol. Lett. 260 (2006) 6976.

[8] H. Maruyama, M. Shin, T. Oda, R. Matsumi, R.L. Ohniwa, T. Itoh, K. Shirahige, T. Imanaka, H. Atomi, S.H. Yoshimura, K. Takeyasu, Histone and TK0471/TrmBL2 form a novel heterogeneous genome architecture in the hyperthermophilic archaeon Thermococcus kodakarensis, Mol. Biol. Cell 22 (2011) 386398.

[9] A.K. Efremov, Y. Qu, H. Maruyama, C.J. Lim, K. Takeyasu, J. Yan, Transcriptional repressor TrmBL2 from Thermococcus kodakarensis forms filamentous nucleoprotein structures and competes with histones for DNA binding in a salt- and DNA supercoiling-dependent manner, J. Biol. Chem. 290 (2015) 1577015784

[10] K. Sandman, J.N. Reeve, Archaeal histones and the origin of the histone fold, Curr. Opin. Microbiol. 9 (2006) 520525.

[11] N. Laurens, R.P. Driessen, I. Heller, D. Vorselen, M.C. Noom, F.J. Hol, M.F. White, R.T. Dame, G.J. Wuite, Alba shapes the archaeal genome using a delicate balance of bridging and stiffening the DNA, Nat. Commun. 3 (2012) 1328.

[12] E. Peeters, R.P. Driessen, F. Werner, R.T. Dame, The interplay between nucleoid organization and transcription in archaeal genomes, Nat. Rev. Microbiol. 13 (2015) 333341.

[13] E. Krissinel, K. Henrick, Secondary-structure matching (SSM), a new tool for fast protein structure alignment in three dimensions, Acta Crystallogr. D Biol. Crystallogr. 60 (2004) 22562268.

[14] P. Emsley, K. Cowtan, Coot: Model-building tools for molecular graphics, Acta Crystallogr. D Biol. Crystallogr. 60 (2004) 21262132.

[15] M. Lewis, G. Chang, N.C. Horton, M.A. Kercher, H.C. Pace, M.A. Schumacher, R.G. Brennan, P. Lu, Crystal structure of the lactose operon repressor and its complexes with DNA and inducer, Science 271 (1996) 12471254.

[16] M.A. Schumacher, K.Y. Choi, H. Zalkin, R.G. Brennan, Crystal structure of Lacl member, PurR, bound to DNA: Minor groove binding by alpha helices, Science 266 (1994) 763770.

[17] W. Hinrichs, C. Kisker, M. Duvel, A. Muller, K. Tovar, W. Hillen, W. Saenger, Structure of the Tet repressor-tetracycline complex and regulation of antibiotic resistance, Science 264 (1994) 418420

[18] R.G. Brennan, B.W. Matthews, The helix turn helix DNA binding motif, J. Biol. Chem. 264 (1989) 19031906.

[19] A. Vangone, R. Spinelli, V. Scarano, L. Cavallo, R. Oliva, COCOMAPS: A Web application to analyze and visualize contacts at the interface of biomolecular complexes, Bioinformatics 27 (2011) 29152916.

[20] D. Lu, S. Fillet, C. Meng, Y. Alguel, P. Kloppsteck, J. Bergeron, T. Krell, M.T. Gallegos, J. Ramos, X. Zhang, Crystal structure of $T \operatorname{tg} V$ in complex with its DNA operator reveals a general model for cooperative DNA binding of tetrameric gene regulators, Genes Dev. 24 (2010) 25562565.

[21] J. Ribeiro, F. Melo, A. Schuller, PDlviz: Analysis and visualization of protein DNA binding interfaces, Bioinformatics 31 (2015) 27512753.

[22] J.A. Stuckey, J.E. Dixon, Crystal structure of a phospholipase D family member, Nat. Struct. Biol. 6 (1999) 278284.

[23] E.V. Koonin, A duplicated catalytic motif in a new superfamily of phosphohydrolases and phospholipid synthases that includes poxvirus envelope proteins, Trends Biochem. Sci. 21 (1996) 242243.

[24] C.P. Ponting, I.D. Kerr, A novel family of phospholipase D homologues that includes phospholipid synthases and putative endonucleases: Identification of duplicated repeats and potential active site residues, Protein Sci. 5 (1996) 914922.

[25] L. Chen, L.R. Chen, X.E. Zhou, Y. Wang, M.A. Kahsai, A.T. Clark, S.P. Edmondson, Z.J. Liu, J.P. Rose, B.C. Wang, E.J. Meehan, J.W. Shriver, The hyperthermophile protein Sso10a is a dimer of winged helix DNA-binding domains linked by an antiparallel coiled coil rod, J. Mol. Biol. 341 (2004) 7391. 
[26] E.E. Heldwein, R.G. Brennan, Crystal structure of the transcription activator BmrR bound to DNA and a drug, Nature 409 (2001) 378382.

[27] S.C. Schultz, G.C. Shields, T.A. Steitz, Crystal structure of a CAP-DNA complex: The DNA is bent by 90 degrees, Science 253 (1991) 10011007.

[28] G.M. Harami, M. Gyimesi, M. Kovacs, From keys to bulldozers: Expanding roles for winged helix domains in nucleic-acid-binding proteins, Trends Biochem. Sci. 38 (2013) 364371.

[29] A. Meinhart, J. Blobel, P. Cramer, An extended winged helix domain in general transcription factor E/IIE alpha, J. Biol. Chem. 278 (2003) 4826748274.

[30] C. Wolberger, R. Campbell, New perch for the winged helix, Nat. Struct. Biol. 7 (2000) 261262

[31] K.S. Gajiwala, H. Chen, F. Cornille, B.P. Roques, W. Reith, B. Mach, S.K. Burley, Structure of the winged-helix protein hRFX1 reveals a new mode of DNA binding, Nature 403 (2000) 916921

[32] R. Amit, A.B. Oppenheim, J. Stavans, Increased bending rigidity of single DNA molecules by H-NS, a temperature and osmolarity sensor, Biophys. J. 84 (2003) 24672473.

[33] Y. Liu, H. Chen, L.J. Kenney, J. Yan, A divalent switch drives H-NS/DNA-binding conformations between stiffening and bridging modes, Genes Dev. 24 (2010) 339344.

[34] H. Shindo, T. Iwaki, R. leda, H. Kurumizaka, C. Ueguchi, T. Mizuno, S. Morikawa, H. Nakamura, H. Kuboniwa, Solution structure of the DNA binding domain of a nucleoid-associated protein, H-NS, from Escherichia coli, FEBS Lett. 360 (1995) 125131.

[35] S.T. Arold, P.G. Leonard, G.N. Parkinson, J.E. Ladbury, HNS forms a superhelical protein scaffold for DNA condensation, Proc. Natl. Acad. Sci. U. S. A. 107 (2010) 1572815732.

[36] Y.G. Gao, S.Y. Su, H. Robinson, S. Padmanabhan, L. Lim, B.S. McCrary, S.P. Edmondson, J.W. Shriver, A.H. Wang, The crystal structure of the hyperthermophile chromosomal protein Sso7d bound to DNA, Nat. Struct. Biol. 5 (1998) 782786.

[37] Z. Zhang, L. Guo, L. Huang, Archaeal chromatin proteins, Sci. China Life Sci. 55 (2012) 377385.

[38] E.L. Cam, F. Culard, E. Larquet, E. Delain, J.A. Cognet, DNA bending induced by the archaebacterial histone-like protein MC1, J. Mol. Biol. 285 (1999) 10111021.

[39] M. Crnigoj, Z. Podlesek, M. Zorko, R. Jerala, G. Anderluh, N.P. Ulrih, Interactions of archaeal chromatin proteins Alba1 and Alba2 with nucleic acids, PLoS One 8 (2013) e58237.

[40] R.J. DeLange, G.R. Green, D.G. Searcy, A histone-like protein (HTa) from Thermoplasma acidophilum. I. Purification and properties, J. Biol. Chem. 256 (1981) 900904.

[41] L. Guo, Y. Feng, Z. Zhang, H. Yao, Y. Luo, J. Wang, L. Huang, Biochemical and structural characterization of Cren7, a novel chromatin protein conserved among Crenarchaea, Nucleic Acids Res. 36 (2008) 11291137.

[42] J.G. McAfee, S.P. Edmondson, I. Zegar, J.W. Shriver, Equilibrium DNA binding of Sac7d protein from the hyperthermophile Sulfolobus acidocaldarius: Fluorescence and circular dichroism studies, Biochemistry 35 (1996) 40344045.

[43] B.S. McCrary, S.P. Edmondson, J.W. Shriver, Hyperthermophile protein folding thermodynamics: Differential scanning calorimetry and chemical denaturation of Sac7d, J. Mol. Biol. 264 (1996) 784805.
[44] I. Waege, G. Schmid, S. Thumann, M. Thomm, W. Hausner, Shuttle vector-based transformation system for Pyrococcus furiosus, Appl. Environ. Microbiol. 76 (2010) 33083313.

[45] B. Sommer, I. Waege, D. Pollmann, T. Seitz, M. Thomm, R. Sterner, W. Hausner, Activation of a chimeric Rpb5/RpoH subunit using library selection, PLoS One 9 (2014) e87485.

[46] W. Kabsch, XDS, Acta Crystallogr. D. Biol. Crystallogr. 66 (2010) 125132.

[47] P.A. Karplus, K. Diederichs, Linking crystallographic model and data quality, Science 336 (2012) 10301033.

[48] P.D. Adams, K. Gopal, R.W. Grosse-Kunstleve, L.W. Hung, T.R. loerger, A.J. McCoy, N.W. Moriarty, R.K. Pai, R.J. Read, T.D. Romo, J.C. Sacchettini, N.K. Sauter, L.C. Storoni, T.C. Terwilliger, Recent developments in the PHENIX software for automated crystallographic structure determination, J. Synchrotron Radiat. 11 (2004) 5355.

[49] P.D. Adams, P.V. Afonine, G. Bunkoczi, V.B. Chen, I.W. Davis, N. Echols, J.J. Headd, L.W. Hung, G.J. Kapral, R.W. Grosse-Kunstleve, A.J. McCoy, N.W. Moriarty, R. Oeffner, R.J. Read, D.C. Richardson, J.S. Richardson, T.C. Terwilliger, P.H. Zwart, PHENIX: A comprehensive Pythonbased system for macromolecular structure solution, Acta Crystallogr. D Biol. Crystallogr. 66 (2010) 213221.

[50] P. Emsley, B. Lohkamp, W.G. Scott, K. Cowtan, Features and development of Coot, Acta Crystallogr. D Biol. Crystallogr. 66 (2010) 486501.

[51] J. Painter, E.A. Merritt, Optimal description of a protein structure in terms of multiple groups undergoing TLS motion, Acta Crystallogr. D Biol. Crystallogr. 62 (2006) 439450.

[52] A.J. McCoy, R.W. Grosse-Kunstleve, P.D. Adams, M.D. Winn, L.C. Storoni, R.J. Read, Phaser crystallographic software, J. Appl. Crystallogr. 40 (2007) 658674.

[53] F. DiMaio, N. Echols, J.J. Headd, T.C. Terwilliger, P.D. Adams, D. Baker, Improved low-resolution crystallographic refinement with Phenix and Rosetta, Nat. Methods 10 (2013) 11021104.

[54] J.M. Duarte, A. Srebniak, M.A. Scharer, G. Capitani, Protein interface classification by evolutionary analysis, BMC Bioinf. 13 (2012) 334.

[55] R. Lavery, M. Moakher, J.H. Maddocks, D. Petkeviciute, K. Zakrzewska, Conformational analysis of nucleic acids revisited: Curves+, Nucleic Acids Res. 37 (2009) 59175929.

[56] W. Kabsch, C. Sander, Dictionary of protein secondary structure: Pattern recognition of hydrogen-bonded and geometrical features, Biopolymers 22 (1983) 25772637.

[57] X. Robert, P. Gouet, Deciphering key features in protein structures with the new ENDscript server, Nucleic Acids Res. 42 (2014) W320 W324.

[58] N.A. Baker, D. Sept, S. Joseph, M.J. Holst, J.A. McCammon, Electrostatics of nanosystems: Application to microtubules and the ribosome, Proc. Natl. Acad. Sci. U. S. A. 98 (2001) 1003710041.

[59] T.J. Dolinsky, J.E. Nielsen, J.A. McCammon, N.A. Baker, PDB2PQR: An automated pipeline for the setup of PoissonBoltzmann electrostatics calculations, Nucleic Acids Res. 32 (2004) W665 W667.

[60] T.J. Dolinsky, P. Czodrowski, H. Li, J.E. Nielsen, J.H. Jensen, G. Klebe, N.A. Baker, PDB2PQR: Expanding and upgrading automated preparation of biomolecular structures for molecular simulations, Nucleic Acids Res. 35 (2007) W522 W525. 